\title{
Provision of home parenteral nutrition in northern England from 2000 to 2006
}

\author{
D. A. Bull ${ }^{1}$, J. Ledger ${ }^{2}$, J. Wilkins ${ }^{2}$, B. Davidson ${ }^{2}$, L. Fletcher ${ }^{2}$, D. McLure ${ }^{2}$ and N. P. Thompson ${ }^{2}$ \\ ${ }^{1}$ Newcastle University Medical School, Framlington Place, Newcastle upon Tyne, UK and ${ }^{2}$ Departments of Medicine, \\ Surgery, Biochemistry and Dietetics, Freeman Hospital, Newcastle upon Tyne NE7 7DN, UK
}

Home parenteral nutrition (HPN) is necessary for those who have long-term gastrointestinal failure such that they are not able to maintain themselves solely with enteral nutrition. In the northern region of England (extending from Cumbria to Tyneside, Wearside and Teeside, with a population of about 2.9 million) there was no organised service for the provision of adult HPN prior to 2000. From this time a multi-disciplinary regional service was developed, based at the Freeman Hospital, Newcastle. This is a retrospective audit of the service from 1 January 2000 to 31 December 2006. Hospital notes were retrieved for all patients.

Thirty-four patients were identified as receiving HPN during this period; one patient transferred their care after a short period to another hospital and was not included (he remains well on HPN). Thirty-three patients (eighteen male) had a mean age of 50 years (range 16-72 years). The most common indications were Crohn's disease (seven), mesenteric infarction (seven), malignancy (seven) and post-surgical complications (six). The total number of HPN days was 26153; median 360 (range 43-2555). Only four patients did not eat at all. The number of patients managed at any one time at the regional centre has increased from one in 2000-2001 to fourteen to eighteen over the last 2 years.

Total number of in-patient hospital days after initial training and discharge was 1838; median 22 (range 0-534) and of these 370 were due to complications of their HPN; median 4 (range 0-52). There were twenty-nine proven catheter infections, which resulted in twentyone catheter changes and an additional eleven possible catheter infections with seven line changes. This amounts to 1.1 definite catheter infections per 1000 HPN days and 1.5 definite or possible catheter infections per 1000 HPN days. There were an additional twenty-one catheter changes due to either catheter occlusion or damage. Six patients developed biliary complications, five requiring Endoscopic Retrograde Cholangiopancreatography (ERCP) with removal of ductal calculi and one having acalculous cholecystitis. Twenty-one patients had Dual Energy X-ray Absorptiometry (DEXA) scans with seven having osteoporosis and eight osteopaenia, using WHO criteria.

At the end of 2006 fourteen patients still required HPN, eleven had ceased to need it and eight had died; five deaths occurred within 6 months of commencing HPN. HPN had only been a factor in the death of one patient who had developed infective endocarditis of a prosthetic heart valve. The 5-year survival on HPN was approximately $55 \%$ and patients had a $38 \%$ chance of becoming independent of PN during this time. 